\title{
Ionic Strength Dependence of the Length of Charged Linear Micelles
}

\author{
Theo Odijk \\ Department of Polymer Technology, Faculty of Chemical Engineering and Materials Science, Delft University \\ of Technology, P.O. Box 5045, 2600 GA Delft. The Netherlands (Received: December 27, 1988)
}

A simple theory is presented for the lengthening of charged micelles with increasing salt concentration. An electrostatic end effect is incorporated in the current theory of micellar aggregation. At low salt concentrations the logarithm of the micellar length should decrease in proportion to the inverse square root of the ionic strength.

The size of a linear micelle is governed by end effects ${ }^{1}$ because the chemical potential of one amphiphile determines the statistical thermodynamics of the aggregating system. This implies that, to a large extent, the properties of linear aggregates cannot be as universal as those of linear macromolecules. Concomitantly, greater care must be exercised in developing approximations for the interactions in micelles. For instance, in polymer theory minor error is incurred on replacing the real potential of mean force between segments in a good solvent with an effective hard core repulsion but whether an analogous substitution works well for micelles is open to doubt. Here, we do not delve into these problems but rather propose a crude explanation for the lengthening of charged micellar rods as the ionic strength increases. If the Debye screening length is substantially longer than both the micellar diameter and the distance between charged surfactant heads, we expect the micelles to adopt fairly universal behavior with regard to the ionic strength dependence of their length.

First we discuss the approximate form of the electrostatic free energy of a linear micelle. The aggregate is replaced by a charged line of length $L$ and linear charge density $q \nu$, where $q$ is the elementary charge and $\nu$ is the number of charges per unit length along the micellar axis. It is immersed in a 1-1 electrolyte solution of concentration $c$. An important electrostatic scale is the Bjerrum length $Q=q^{2} / D k_{\mathrm{B}} T$, where $D$ is the uniform permittivity of the solvent, $k_{\mathrm{B}}$ is Boltzmann's constant, and $T$ is the temperature. In terms of $Q$ the Debye length $\kappa^{-1}$ is given by $\kappa^{2}=8 \pi Q c$ provided the salt swamps any counterions arising from the surfactant. The electrostatic free energy $f$ is now assumed to be well represented by a superposition of Debye-Hückel potentials

$$
\begin{gathered}
f / k_{\mathrm{B}} T=\nu^{2} Q \int_{\epsilon}^{L} \mathrm{~d} t(L-t) t^{-1} e^{-\kappa t} \\
=\nu^{2} Q\left[L \operatorname{Ei}(\kappa \epsilon)-L \operatorname{Ei}(\kappa L)-\kappa^{-1}\left(e^{-\kappa \epsilon}-e^{-\kappa L}\right)\right]
\end{gathered}
$$

where Ei is exponential integral. We have here had to introduce a short distance cutoff $\epsilon$; otherwise $f$ would diverge. For the real micelle $\epsilon$ should be of the order of its diameter or charge spacing. The dependence on $\epsilon$ may seem worrisome, but it turns out that the micellar length becomes independent of $\epsilon$ as $\epsilon \kappa$ tends to zero.

Equation 1 is satisfactory when the charge spacing $y^{-1}$ is big enough $-\nu^{-1} \gg Q$, say - as long as $\kappa \epsilon$ is much smaller than unity so that discrete charge effects may be neglected. Nevertheless, in practice the charge density can be quite high, causing the (apparent) invalidity of the Debye-Hückel approximation. In that case counterions accumulate around each micelle, nonlinear screening occurs, and $f$ needs to be evaluated via the PoissonBoltzmann equation, for instance. However, it is a common and useful approximation to adopt eq 1 even when $\nu \gtrsim Q^{-1}$ but with an effective charge density $\nu_{\text {eff }} \simeq Q^{-1}$. In the present calculation this procedure may seem dubious since our aim is to investigate end effects. Thus, we might expect $\nu_{\text {eff }}$ to be nonuniform, i.e., $\nu_{\text {eff }}$ $\simeq Q^{-1}$ in the midsection of the micelle but $\nu_{\text {eff }} \simeq \nu$ for the end sections of length $\kappa^{-1}$ when $\kappa L$ is much greater than unity. Fortunately, the approximation $\nu_{\text {eff }} \simeq Q^{-1}$ is quite good even at the

(1) Israelachvili, J. N.; Mitchell, D. J.; Ninham, B. W. J. Chem. Soc., Faraday Trans. 2 1976, $72,1525$. ends in view of elaborate work by Ramanathan and Woodbury. They were able to prove the following result for short polyelectrolyte rods $(\kappa L=\mathcal{O}(1))$ that are highly charged

$$
\nu_{\text {eff }} \simeq \frac{\ln \kappa a}{Q \ln (a / L)}
$$

which is near the usual approximation $\nu_{\text {eff }} \simeq Q^{-1}$ valid for long rods $(\kappa L \gg 1)$. This expression implies that end effects on the effective charge density may be disregarded to a first approximation for both short and long rods. Hence, eq 1 neglects only logarithmic terms when the micelle is highly charged provided we choose an effective charge spacing instead of the bare one. The following result needed below is readily derived from eq $1:$

$$
\mathrm{d}\left(f / L k_{\mathrm{B}} T\right) / \mathrm{d} L=\frac{\nu^{2} Q}{L^{2} \kappa}\left(e^{-\kappa \epsilon}-e^{-\kappa L}\right)
$$

Note that this expression is well-defined even when $\epsilon$ is set equal to zero. Moreover, it is a monotone decreasing function as $k$ increases.

For the purpose of our calculation the effect of micellar polydispersity is of little interest so we conveniently force the aggregates to be monodisperse. The system is viewed as a dilute solution of noninteracting slender rodlike micelles each consisting of $s$ amphiphiles. The number $s$ is as yet unknown and is regarded as a variational parameter. The chemical potential $\mu$ of one amphiphile is given by the usual expression ${ }^{1,3,4}$ except for the present inclusion of the screened electrostatic interaction.

$$
\mu=\mu_{0}+K s^{-1}+s^{-1} \ln \varphi-n s^{-1} \ln s+y(s)
$$

The constant $\mu_{0}$ arises from the "extensive" interactions within the main body of every micelle whereas the other terms may be denoted by end effects. The constant $K$ pertaining to the micellar caps is left unspecified. One can discern a mixing contribution depending on the micellar volume fraction $\varphi$. Concomitantly, a term $-s^{-1}$ ln $s$ also stems from the entropy of mixing as was discussed in ref 1 . If the micelles grow for whatever reason, the number of translational and rotational degrees of freedom in the system should decrease because the total number of amphiphiles is fixed. Considerations ${ }^{3,4}$ analogous to those in the theory of ideal gases show that $n=1$ (mixing) $+3 / 2$ (trans) $+7 / 2$ (rot) $=6$. The electrostatic term $y(s)=f / s k_{\mathrm{B}} T$ is new and its influence will now be discussed. Note that $K$ is assumed to be independent of the ionic strength, a supposition that is reasonable presumably for low ionic strength when $\kappa \epsilon \ll 1$.

In order to find the micellar length, we simply minimize the total free energy or equivalently the chemical potential $\mu$ with respect to the number of surfactant molecules $s$. We remark that the ratio $z=L / s$ is a constant for the slender micelles we are dealing with. Equations 2 and 3 lead to the solution

(2) Ramanathan, G. V.; Woodbury, Jr., C. P. J. Chem. Phys. 1982, 77, 4133

(3) Nagarajan, R.; Ruckenstein, E. J. Colloid Interface Sci. 1979, 71, 580

(4) McMullen III, W. E.; Gelbart, W. M.; Ben-Shaul, A. J. Phys, Chem. $1984,88,6649$ 


$$
n \ln s_{\mathrm{m}}=K+\ln \varphi+n-\nu^{2} Q \kappa^{-1}\left(e^{-\mathrm{\alpha t}}-\epsilon^{-\mathrm{\alpha} s_{\mathrm{m}}}\right)
$$

It is immediately clear that the micellar length increases with increasing salt concentration and the electrostatic end effect can easily compete with the usual terms.

When comparing this calculation with experiment, it is convenient to scale the charge density as $\nu_{*}=\nu Q$. Furthermore, if the ionic strength is such that $\kappa \epsilon \ll 1 \ll \kappa L$, it is expedient to rewrite eq 4 as

$$
\ln L=-\frac{\nu_{*}^{2}}{n Q_{\kappa}}+\text { constant }
$$

Unfortunately, in most experiments the ionic strength is so high that $\kappa \epsilon=\mathscr{O}(1)$ and our naive treatment breaks down. Hoffmann et al. ${ }^{5}$ did perform small-angle neutron scattering on cetylpyridinyl salicylate solutions in $\mathrm{D}_{2} \mathrm{O}$ at fairly low $\mathrm{NaCl}$ concentrations. The semiflexibility of the wormlike micelles turned out to be slight so we may neglect a possible change in $n$. Table I shows the variation of the contour length with $\mathrm{NaCl}$ concentration, the volume fraction $\varphi$ remaining fixed. Since the micelles are about $5 \mathrm{~nm}$ thick, the quantity $\kappa \in$ is already greater than unity at an

(5) Hoffmann, H.; Kalus, J.; Thurn, H.; Ibel, K. Ber. Bunsen-Ges. Phys. Chem. 1983, 87, 1120 .
TABLE I: Micellar Length $L$ as a Function of the $\mathrm{NaCl}$ Concentration for Cetylpyridinyl Salicylate in $\mathrm{D}_{2} \mathrm{O}$

$\begin{array}{lllll}c,{ }^{a} \mathrm{mM} & 0.5 & 2 & 8 & 32 \\ (\kappa Q)^{-1 b} & 18.9 & 9.4 & 4.8 & 2.36 \\ L,{ }^{a} \mathrm{~nm} & 55 & 75 & 100 & 300 \\ \ln L & 6.3 & 6.6 & 6.9 & 8.0\end{array}$

${ }^{a}$ Data from ref 5. ${ }^{b}$ The Bjerrum length $Q$ equals $0.717 \mathrm{~nm}$ for $\mathrm{D}_{2} \mathrm{O}$ at $298 \mathrm{~K}$

ionic strength of $8 \mathrm{mM}$. Accordingly, the theory must be suspect at this and higher salt concentrations. Moreover, there is a good deal of uncertainty in the experimental values of the contour lengths. ${ }^{5}$ Therefore, even in this experiment it is not possible to refute or verify our contention that at low ionic strength the logarithm of the micellar length should be proportional to the inverse of $k Q$.

Nevertheless, at low ionic strength eq 5 does predict the right magnitude for the change in the micellar contour. In fact, the entries for the two lowest salt concentrations in Table I wr'sld impose a scaled charge density $\nu_{*}$ equal to about 0.5 if they are to agree with eq 5 (setting $n$ equal to 6). This corresponds to a charge spacing of $1.5 \mathrm{~nm}$, close to the estimate given by Hoffmann et al. ${ }^{5}$ Accordingly, it would be interesting to check eq 5 for solutions of linear micelles at suitably chosen ionic strengths.

\title{
Observation of High-Energy Backward Scattered Ions In a Beam Study of Threshold Energy Collision-Induced Dlssoclation: Dynamics of a Long-Lived Exclted State of the Acetone Molecular Ion
}

\author{
Kuangnan Qian, Anil Shukla,* Stephen Howard, Stephen Anderson, and Jean Futrell \\ Department of Chemistry and Biochemistry, University of Delaware, Newark, Delaware 19716 \\ (Received: January 25, 1989)
}

\begin{abstract}
Collision-induced dissociation of acetone molecular ions with helium as a target gas has been studied at $0.65-$ and $0.45-\mathrm{eV}$ center-of-mass collision energies. Scattering contour maps show that fragment ions, $\mathrm{CH}_{3} \mathrm{CO}^{+}$, are predominantly backward scattered with intensity maxima lying well outside the elastic scattering circle. This can occur only by the conversion of internal energy into translational energy, demonstrating the presence of long-lived $(\tau>30 \mu \mathrm{s})$ excited states of the acetone molecular ion. From the observed reaction dynamics we infer that the first electronically excited state of acetone ion has the unique characteristic that on collision it releases all the excess energy into the recoil kinetic energy of the resulting internally excited ground state ion which then rapidly fragments into $\mathrm{CH}_{3} \mathrm{CO}^{+}$and $\mathrm{CH}_{3}$.
\end{abstract}

\section{Introduction}

In a recent study of the collision-induced dissociation (CID) of acetone ion to acetyl ion we observed two dynamically distinct mechanisms. ${ }^{1}$ Backward scattering of fragment ions is the dominant mechanism at low energy (1.9-eV center of mass); at higher energies forward scattering with intensity maxima at nonzero angles is the dominant process. Both mechanisms are translationally endoergic; kinetic energy well in excess of that required by the thermochemical threshold is transferred into internal excitation of the fragmenting acetone ion. In agreement with the high-energy experiments of van der Zande et al., ${ }^{2}$ it was suggested that low impact parameter collisions initiate the CID of acetone ion at all energies.

We have now extended our study of acetone ion CID to even lower energies near and below the thermochemical threshold in

(1) Shukla, A. K.; Qian, K.; Howard, S. L.; Anderson, S. G.; Futrell, J. H. Int. J. Mass Spectrom. Ion Processes, in press.

(2) van der Zande, W. J.; de Bruijn, D. P.; Loss, J.; Kistemaker, P. G.; McLuckey, S. A. Int. J. Mass Spectrom. Ion Processes 1985, 67, 161. order to understand its dissociation dynamics and better define energy transfer in CID. In this Letter we present these data demonstrating the unanticipated result that the first electronically excited state of acetone ion (A state) is long-lived and exhibits unique fragmentation characteristics.

\section{Experimental Section}

The apparatus used for the present study has been described in detail in an earlier publication. ${ }^{3}$ Briefly, 3-keV acetone ions generated by electron impact ionization and mass selected by a Vacuum Generators VG 7070E double-focusing mass spectrometer were decelerated to the desired energy at the collision center by a series of cylindrical and rectangular tube lenses. The decelerated ion beam then collided with a vertically moving (upward directed) supersonic beam of neutrals, and resulting acetyl fragment ions $(\mathrm{m} / \mathrm{z} 43)$ were energy and mass analyzed by a hemispherical energy analyzer and a quadrupole mass filter at a series of lab-

(3) Shukla, A. K.; Anderson, S. G.; Howard, S. L.; Sohlberg, K. W. Futrell, J. H. Int. J. Mass Spectrom. Ion Processes 1988, 86, 61 . 